\title{
Generation of Carbamoyl- and Thiocarbamoyllithium Synthons Having a Hydrogen(s) or an Aryl Group on the Nitrogen and Their Trapping with Electrophiles
}

\author{
Nobuaki Kambe, ${ }^{*}$ Toru Inoue, Takanobu Takeda, Shin-ichi Fujiwara, and Noboru Sonoda ${ }^{\S}$ \\ Department of Applied Chemistry, Graduate School of Engineering, Osaka University, \\ Suita, Osaka 565-0871, Japan
}

\section{Supporting Information}

1) General Comments

2) Confirmation of the Formation of Aluminum Azaenolate of Carbamotelluroate 3a

3) Typical Procedure for Conversion of Isocyanates to Carbamoyllithium Synthons and Characterization Data of 6a-g

4) Dearylation of $6 \mathrm{~g}$ with $\mathrm{CAN}$

5) General Procedure for Conversion of Isothiocyanates to Thiocarbamoyllithium Synthons and Characterization Data of 8a-e

6) References

\section{1) General Comment.}

${ }^{n} \mathrm{BuLi}$ (1.6 $\mathrm{M}$ in ${ }^{n}$ hexane) and ${ }^{i} \mathrm{Bu}_{2} \mathrm{AlH}$ (1 $\mathrm{M}$ in ${ }^{n}$ hexane) were purchased from Kanto Chemical CO., Inc. Dibutyl ditelluride was synthesized by air oxidation of ${ }^{n} \mathrm{BuTeLi}$, prepared by the reaction of ${ }^{n} \mathrm{BuLi}$ with equimolar amounts of metallic tellurium. ${ }^{\mathrm{s}}{ }^{1}$

Isocyanates and isothiocyanates are commercially available and used without further purification. Electrophiles were distilled prior to use. THF and $\mathrm{Et}_{2} \mathrm{O}$ were distilled from benzophenone ketyl immediately before use. Silica gel (wakogel B-5F) was used for preparative thin layer chromatography. HPLC separations were performed on a recycling preparative HPLC (Japan Analytical Industry Co. Ltd., Model LC-908), equipped with JAIGEL-lH and $2 \mathrm{H}$ columns (GPC) using $\mathrm{CHCl}_{3}$ as an eluent. Melting points (uncorrected) were determined by using a Yanagimoto micro melting point apparatus. ${ }^{1} \mathrm{H}$ and ${ }^{13} \mathrm{C}$ NMR spectra were recorded on a JEOL JNM-GSX-270 spectrometer using $\mathrm{CDCl}_{3}$ as a solvent with $\mathrm{Me}_{4} \mathrm{Si}$ as an internal standard. IR spectra were obtained on a Perkin-Elmer Model 1600 spectrometer. Mass spectra were measured on a Hitachi Model RMU-6E. Elemental analyses were performed on a Yanagimoto CHN-Corder MT-2. 
2) Confirmation of Formation of Aluminum Azaenolate of Carbamotelluroate 3a. To a flame dried flask equipped with an Ar inlet and a rubber septum were placed ${ }^{n} \mathrm{Bu}_{2} \mathrm{Te}_{2}(185 \mathrm{mg}, 0.50 \mathrm{mmol})$ and ${ }^{i} \mathrm{Bu}_{2} \mathrm{AlH}\left(1 \mathrm{M}\right.$ in ${ }^{n}$ hexane, $\left.1.0 \mathrm{~mL}, 1 \mathrm{mmol}\right)$ under an argon atmosphere. The solution was stirred at $25{ }^{\circ} \mathrm{C}$ for $1 \mathrm{~h}$ and cooled to $-23{ }^{\circ} \mathrm{C}$. After $2 \mathrm{~mL}$ of THF was added, $1 \mathrm{mmol}$ of butyl isocyanate $(99 \mathrm{mg})$ was injected. The resulting mixture was stirred at $-23{ }^{\circ} \mathrm{C}$ for $10 \mathrm{~min}$, then gradually warmed up to $0{ }^{\circ} \mathrm{C}$ and maintained at the temperature for $1 \mathrm{~h}$. The solution was poured into a saturated aqueous $\mathrm{NH}_{4} \mathrm{Cl}$ solution. Products were extracted with $\mathrm{Et}_{2} \mathrm{O}$ (30 mL x 3), dried over $\mathrm{MgSO}_{4}$, and concentrated in vacuo. Short column chromatography ( ${ }^{n}$ hexane then $\mathrm{Et}_{2} \mathrm{O}$ ) of the residue gave pure 4a $(250 \mathrm{mg}, 88 \%)$ in the $\mathrm{Et}_{2} \mathrm{O}$ fraction: ${ }^{1} \mathrm{H} \mathrm{NMR}(270 \mathrm{MHz}$, $\left.\mathrm{CDCl}_{3}\right) \delta 0.92(\mathrm{t}, J=7.3 \mathrm{~Hz}, 6 \mathrm{H}), 1.37-1.46(\mathrm{~m}, 4 \mathrm{H}) 1.52$ (quint, $J=7.3 \mathrm{~Hz}, 2 \mathrm{H}$ ), 1.85 (quint, $J=7.3 \mathrm{~Hz}, 2 \mathrm{H}), 2.95(\mathrm{t}, J=7.3 \mathrm{~Hz}, 2 \mathrm{H}), 3.34$ (q, $J=7.3 \mathrm{~Hz}, 2 \mathrm{H}), 6.24$ (s, $1 \mathrm{H}) ;{ }^{13} \mathrm{C}$ NMR $\left(68 \mathrm{MHz} \mathrm{CDCl}_{3}\right) \delta 1.13\left(J_{\mathrm{C}-\mathrm{Te}}=74.2 \mathrm{~Hz}\right), 13.2,13.5,19.8,25.1,31.5$, 34.3, 41.5, 150.3; IR (neat) 3307, 2958, 2930, 1643, $1204 \mathrm{~cm}^{-1}$; MS m/z (relative intensity) $287\left(\mathrm{M}^{+} 13\right), 188$ (30), 100 (14) 57 (100); HRMS calcd for $\mathrm{C}_{9} \mathrm{H}_{19} \mathrm{NOTe}$ 287.0529, found 287.0515.

3) Typical Procedure for Conversion of Isocyanates to Carbamoyllithium Synthons. A mixture of ${ }^{n} \mathrm{Bu}_{2} \mathrm{Te}_{2}(185 \mathrm{mg}, 0.50 \mathrm{mmol})$ and ${ }^{i} \mathrm{Bu}_{2} \mathrm{AlH}\left(1 \mathrm{M}\right.$ in ${ }^{n}$ hexane, $1.0 \mathrm{~mL}, 1$ mmol) was stirred at $25{ }^{\circ} \mathrm{C}$ for $1 \mathrm{~h}$. After the solution was cooled to $-23{ }^{\circ} \mathrm{C}, 2 \mathrm{~mL}$ of THF and then $1 \mathrm{mmol}$ of butyl isocyanate $(99 \mathrm{mg})$ were added. The resulting mixture was maintained at $-23{ }^{\circ} \mathrm{C}$ for $10 \mathrm{~min}$ and gradually warmed up to $0{ }^{\circ} \mathrm{C}$. Stirring for additional $1 \mathrm{~h}, 3 \mathrm{~mL}$ of THF and $4 \mathrm{~mL}$ of $\mathrm{Et}_{2} \mathrm{O}$ were added to the mixture and then was cooled to $-105{ }^{\circ} \mathrm{C}$. The tellurium-lithium exchange reaction was conducted by using $0.69 \mathrm{~mL}$ of ${ }^{n} \mathrm{BuLi}\left(1.6 \mathrm{M}\right.$ in ${ }^{n}$ hexane, $\left.1.1 \mathrm{mmol}\right)$ and after stirring for $30 \mathrm{~min}$, benzaldehyde (159 mg, $1.5 \mathrm{mmol}$ ) was added then gradually warmed up to $25^{\circ} \mathrm{C}$. After $2 \mathrm{~h}$, the mixture was poured into a saturated aqueous $\mathrm{NH}_{4} \mathrm{Cl}$ solution and extracted with $\mathrm{Et}_{2} \mathrm{O}(30 \mathrm{~mL} \times 3)$. The solvent was evaporated in vacuo and the residue was chromathographed on a short column to remove tellurides ( ${ }^{n}$ hexane then $\left.\mathrm{Et}_{2} \mathrm{O}\right)$. The products in the $\mathrm{Et}_{2} \mathrm{O}$ fraction were separated by PTLC ( ${ }^{n}{ }^{h}$ exane/ $\left./ \mathrm{Et}_{2} \mathrm{O}=10 / 1\right)$ and $\boldsymbol{N}$-butyl- $\alpha$-hydroxybenzeneacetamide (6a) was obtained as white solid (168 $\mathrm{mg}$, 81\%): mp 52.0-53.0 ${ }^{\circ} \mathrm{C}$; ${ }^{1} \mathrm{H}$ NMR $\left(270 \mathrm{MHz}, \mathrm{CDCl}_{3}\right) \delta 0.87(\mathrm{t}, J=7.3 \mathrm{~Hz}, 3 \mathrm{H})$, 1.21-1.44 (m, $4 \mathrm{H}), 3.17$ (q, J = 7.3 Hz, $2 \mathrm{H}), 4.39$ (s, $1 \mathrm{H}), 4.91$ (s, $1 \mathrm{H}), 6.53$ (s, $1 \mathrm{H})$, 7.29-7.33 (m, $5 \mathrm{H}) ;{ }^{13} \mathrm{C}$ NMR $\left(68 \mathrm{MHz}, \mathrm{CDCl}_{3}\right) \delta 13.6,19.9,31.4,39.1,74.0,126.7$, 128.3, 128.6, 139.7, 172.3; IR (KBr) 3295, 2958, 2932, 1642, $1542 \mathrm{~cm}^{-1} ; \mathrm{MS} \mathrm{m} / \mathrm{z}$ (relative intensity) $207\left(\mathrm{M}^{+}, 1.9\right), 108$ (100), 107 (94), 79 (72), 57 (56), Anal. Calcd for $\mathrm{C}_{12} \mathrm{H}_{17} \mathrm{NO}_{2}$ : C, 69.53; H, 8.27; N, 6.76. Found: C, 69.56; H, 8.38; N, 6.66.

$N$-Butyl- $\alpha$-hydroxy- $\alpha, \alpha$-pentamethyleneacetamide (6b). Isolation was performed by recrystallization from ${ }^{n}$ hexane. Mp 96.0-97.0 ${ }^{\circ} \mathrm{C} ;{ }^{1} \mathrm{H}$ NMR $\left(270 \mathrm{MHz}, \mathrm{CDCl}_{3}\right) \delta$ $0.92(\mathrm{t}, J=6.8 \mathrm{~Hz}, 3 \mathrm{H}), 1.26-1.72(\mathrm{~m}, 12 \mathrm{H}), 1.82-1.95(\mathrm{~m}, 2 \mathrm{H}), 3.07$ (d, $J=11.7 \mathrm{~Hz}$, $1 \mathrm{H}), 3.23$ (q, $J=6.8 \mathrm{~Hz}, 2 \mathrm{H}), 6.94(\mathrm{~s}, 1 \mathrm{H}) ;{ }^{13} \mathrm{C} \mathrm{NMR}\left(68 \mathrm{MHz}, \mathrm{CDCl}_{3}\right) \delta 13.7,20.0$, 
21.1, 25.0, 31.6, 34.2, 38.8, 74.9, 176.7; IR (KBr) 3314, 2934, 1643, 1548, 1162, 986 $\mathrm{cm}^{-1}$; MS m/z (relative intensity) $199\left(\mathrm{M}^{+}, 4\right), 99$ (100), 81 (48), 73 (19); HRMS calcd for $\mathrm{C}_{11} \mathrm{H}_{21} \mathrm{NO}_{2}$ 199.1572, found 199.1569.

$\boldsymbol{N}$-Butyl- $\boldsymbol{N}$ '-phenyloxamide (6c). Isolation was performed by recrystallization from ${ }^{n}$ hexane. Mp 149.0-151.0 ${ }^{\circ} \mathrm{C} ;{ }^{1} \mathrm{H}$ NMR $\left(270 \mathrm{MHz}, \mathrm{CDC1}_{3}\right) \delta 0.95(\mathrm{t}, J=7.3 \mathrm{~Hz}, 3 \mathrm{H})$, 1.40 (sextet, $J=7.3 \mathrm{~Hz}, 2 \mathrm{H}$ ), 1.59 (quint, $J=7.3 \mathrm{~Hz}, 2 \mathrm{H}$ ), 3.38 (q, $J=7.3 \mathrm{~Hz}, 2 \mathrm{H}$ ), 7.18 (t, $J=7.8 \mathrm{~Hz}, 1 \mathrm{H}), 7.37$ (t, $J=7.8 \mathrm{~Hz}, 2 \mathrm{H}), 7.65$ (d, $J=7.8 \mathrm{~Hz}, 2 \mathrm{H}), 7.66$ (s, 1 $\mathrm{H}), 9.7(\mathrm{~s}, 1 \mathrm{H}) ;{ }^{13} \mathrm{C} \mathrm{NMR}\left(68 \mathrm{MHz}, \mathrm{CDCl}_{3}\right) \delta 13.6,20.0,31.2,39.7,119.8,125.3,129.1$, 136.4, 157.6, 159.9; IR (KBr) 3342, 3273, 2961, 1660, 1527, $1446 \mathrm{~cm}^{-1} ; \mathrm{MS} \mathrm{m} / \mathrm{z}$ (relative intensity) $220\left(\mathrm{M}^{+}, 100\right), 121$ (24), 100 (19), 93 (59), 57 (52); HRMS calcd for $\mathrm{C}_{12} \mathrm{H}_{16} \mathrm{~N}_{2} \mathrm{O}_{2} 220.1212$, found 220.1196 .

$N$-Butylbenzoylformamide (6d). Isolation was performed by PTLC $\left({ }^{n}\right.$ hexane/Et $\left.2 \mathrm{O}=5 / 1\right) .{ }^{1} \mathrm{H} \mathrm{NMR}\left(270 \mathrm{MHz}, \mathrm{CDCl}_{3}\right) \delta 0.96(\mathrm{t}, J=7.5 \mathrm{~Hz}, 3 \mathrm{H}), 1.41$ (sextet, $J=7.5 \mathrm{~Hz}, 2 \mathrm{H}$ ), 1.60 (quint, $J=7.5 \mathrm{~Hz}, 2 \mathrm{H}$ ), 3.40 (q, $J=7.5 \mathrm{~Hz}, 2 \mathrm{H}$ ), 7.11 (s, $1 \mathrm{H}), 7.47$ (t, $J=7.6 \mathrm{~Hz}, 2 \mathrm{H}), 7.62(\mathrm{t}, J=7.6 \mathrm{~Hz}, 1 \mathrm{H}), 8.34(\mathrm{~d}, J=7.6 \mathrm{~Hz}, 2 \mathrm{H}) ;{ }^{13} \mathrm{C}$ NMR $\left(68 \mathrm{MHz}, \mathrm{CDC1}_{3}\right) \delta 13.7,20.0,31.3,39.1,128.4,131.2,133.4,134.3,161.7$, 187.9; IR (neat) 3306, 2959, 2933, 1665, $1221 \mathrm{~cm}^{-1} ; \mathrm{MS} \mathrm{m} / \mathrm{z}$ (relative intensity) $205\left(\mathrm{M}^{+}\right.$, 24), 105 (100), 77 (23), 57 (20); HRMS calcd for $\mathrm{C}_{12} \mathrm{H}_{15} \mathrm{NO}_{2}$ 205.1103, found 205.1109.

$N$-Phenyl- $\alpha$-hydroxybenzeneacetamide (6e). Isolation was performed by recrystallization from $\mathrm{Et}_{2} \mathrm{O} . \mathrm{Mp} 149.0-151.0{ }^{\circ} \mathrm{C} ;{ }^{1} \mathrm{H} \mathrm{NMR}\left(270 \mathrm{MHz}, \mathrm{CDCl}_{3}\right) \delta 3.54(\mathrm{~d}$, $J=3.4 \mathrm{~Hz}, 1 \mathrm{H}), 5.16(\mathrm{~d}, J=3.4 \mathrm{~Hz}, 1 \mathrm{H}), 7.12(\mathrm{t}, J=7.3 \mathrm{~Hz}, 1 \mathrm{H}), 7.26-7.53(\mathrm{~m}, 9 \mathrm{H})$, $8.19(\mathrm{~s}, 1 \mathrm{H}) ;{ }^{13} \mathrm{C}$ NMR $\left(68 \mathrm{MHz}, \mathrm{CDCl}_{3}\right) \delta 74.7,119.8,124.7,126.9,128.91,128.98$, 129.04, 137.0, 139.0, 170.0; IR (KBr) 3301, 3064, 1652, 1602, 1556, 1448, 754, 693 $\mathrm{cm}^{-1}$; MS m/z (relative intensity) $227\left(\mathrm{M}^{+}, 39\right), 121$ (42), 107 (100), 93 (76), 79 (47); HRMS calcd for $\mathrm{C}_{14} \mathrm{H}_{13} \mathrm{NO}_{2} 227.0946$, found 227.0936.

$N$-Phenyl- $\alpha$-hydroxy- $\alpha, \alpha$-pentamethyleneacetamide (6f). Isolation was performed by recrystallization from $\mathrm{Et}_{2} \mathrm{O}$. Mp 170.0-171.0 ${ }^{\circ} \mathrm{C} ;{ }^{1} \mathrm{H}$ NMR $\left(270 \mathrm{MHz}, \mathrm{CDCl}_{3}\right) \delta$ 1.26-1.40 (m, $1 \mathrm{H}), 1.52-1.74(\mathrm{~m}, 7 \mathrm{H}), 1.98-2.09(\mathrm{~m}, 2 \mathrm{H}), 2.40(\mathrm{~s}, 1 \mathrm{H}), 7.10$ (t, $J=7.6$ $\mathrm{Hz}, 1 \mathrm{H}), 7.32$ (t, $J=7.6 \mathrm{~Hz}, 2 \mathrm{H}), 7.57$ (d, $J=7.6 \mathrm{~Hz}, 2 \mathrm{H}), 8.75(\mathrm{~s}, 1 \mathrm{H}) ;{ }^{13} \mathrm{C}$ NMR $(68$ $\left.\mathrm{MHz}, \mathrm{CDCl}_{3}\right) \delta 21.1,24.9,34.1,75.7,119.6,124.2,129.0,137.6,174.5$; IR (KBr) 3326, $1655,1605,1596,1556,1447 \mathrm{~cm}^{-1}$; MS m/z (relative intensity) $219\left(\mathrm{M}^{+}, 45\right), 121$ (55), 99 (96), 93 (100), 81 (51); HRMS calcd for $\mathrm{C}_{13} \mathrm{H}_{17} \mathrm{NO}_{2} 219.1260$, found 219.1255.

$\mathrm{N}$-(4-Methoxyphenyl)- $\alpha$-hydroxybenzeneacetamide (6g). Isolation was performed by recrystallization from $\mathrm{Et}_{2} \mathrm{O} . \mathrm{Mp} 132.5-134.0{ }^{\circ} \mathrm{C} ;{ }^{1} \mathrm{H} \mathrm{NMR}\left(270 \mathrm{MHz}, \mathrm{CDCl}_{3}\right) \delta 3.55$ (s, $1 \mathrm{H}), 3.77(\mathrm{~s}, 3 \mathrm{H}), 5.14(\mathrm{~d}, J=2.9 \mathrm{~Hz}, 1 \mathrm{H}), 6.84(\mathrm{~d}, J=8.8 \mathrm{~Hz}, 2 \mathrm{H}), 7.39-7.48$ (m, $7 \mathrm{H}), 8.05$ (s, $1 \mathrm{H}) ;{ }^{13} \mathrm{C} \mathrm{NMR}\left(68 \mathrm{MHz}, \mathrm{CDCl}_{3}\right) \delta 55.5,74.6,114.2,121.6,126.9,128.8$, 129.0, 130.2, 139.1, 156.7, 169.7; IR (KBr) 3320, 3119, 1647, 1553, 1513, 1262, 841 
$\mathrm{cm}^{-1} ; \mathrm{MS} \mathrm{m} / \mathrm{z}$ (relative intensity) $257\left(\mathrm{M}^{+}, 100\right), 151$ (36), 123 (48), 108 (82), 107 (75), 79 (39); HRMS calcd for $\mathrm{C}_{15} \mathrm{H}_{15} \mathrm{NO}_{3} 257.1052$, found 257.1050 .

4) Dearylation of $6 \mathbf{g}$ with CAN. Reactions of amides $6 \mathbf{g}$ with CAN was conducted according to the literature procedure. ${ }^{\mathrm{S} 2}$

$\alpha$-Hydroxybenzeneacetamide (7). Mp 132.5-134.0 ${ }^{\circ} \mathrm{C}$ [lit. $\left.{ }^{\mathrm{S} 3} \mathrm{mp} 134-135{ }^{\circ} \mathrm{C}\right] ;{ }^{1} \mathrm{H}$ $\operatorname{NMR}\left(270 \mathrm{MHz}, \mathrm{CDCl}_{3}\right) \delta 5.02(\mathrm{~d}, J=3.6 \mathrm{~Hz}, 1 \mathrm{H}), 5.08(\mathrm{~d}, J=3.6 \mathrm{~Hz}, 1 \mathrm{H}), 6.56(\mathrm{~s}$, $1 \mathrm{H}), 7.05(\mathrm{~s}, 1 \mathrm{H}), 7.24-7.36(\mathrm{~m}, 3 \mathrm{H}), 7.49(\mathrm{~d}, J=7.8 \mathrm{~Hz}, 2 \mathrm{H}) ;{ }^{13} \mathrm{C}$ NMR $(68 \mathrm{MHz}$, $\mathrm{CDCl}_{3}$ ). $\delta 74.6,127.5,128.4,128.9,142.2$. 175.2; IR (KBr) 3408, 3260, 1644, 1062 $\mathrm{cm}^{-1} ; \mathrm{MS} \mathrm{m} / \mathrm{z}$ (relative intensity) $151\left(\mathrm{M}^{+}, 8\right), 107$ (100), 79 (46), 77 (30).

5) Conversion of Isothiocyanates to Thiocarbamoyllithium Synthons. A mixture of ${ }^{n} \mathrm{Bu}_{2} \mathrm{Te}_{2}(185 \mathrm{mg}, 0.50 \mathrm{mmol})$ and ${ }^{i} \mathrm{Bu}_{2} \mathrm{AlH}\left(1 \mathrm{M}\right.$ in ${ }^{n}$ hexane, $\left.1.0 \mathrm{~mL}, 1 \mathrm{mmol}\right)$ was stirred at $25{ }^{\circ} \mathrm{C}$ for $1 \mathrm{~h}$. After the solution was cooled to $-23{ }^{\circ} \mathrm{C}, 2 \mathrm{~mL}$ of THF then 1 mmol of isothiocyanate was injected. The resulting mixture was maintained at $-23{ }^{\circ} \mathrm{C}$ for $10 \mathrm{~min}$ and gradually warmed up to $25^{\circ} \mathrm{C}$. After stirring for $2 \mathrm{~h}, 3 \mathrm{~mL}$ of THF and 4 $\mathrm{mL}$ of $\mathrm{Et}_{2} \mathrm{O}$ were added. The solution was cooled to $-105^{\circ} \mathrm{C}$ and $0.69 \mathrm{~mL}$ of ${ }^{n} \mathrm{BuLi}(1.6$ $\mathrm{M}$ in ${ }^{n}$ hexane, $1.1 \mathrm{mmol}$ ) was injected. After $30 \mathrm{~min}, 1.5 \mathrm{mmol}$ of electrophile was added and gradually warmed up to $25{ }^{\circ} \mathrm{C}$. After $2 \mathrm{~h}$, the mixture was poured into a saturated $\mathrm{NH}_{4} \mathrm{Cl}$ solution and extracted with $\mathrm{Et}_{2} \mathrm{O}(30 \mathrm{~mL}$ x 3). The solvent was evaporated in vacuo and the residue was chromatographed on a short column to remove tellurides ( ${ }^{n}$ hexane then $\mathrm{Et}_{2} \mathrm{O}$ ). Isolation was conducted as described below.

$\boldsymbol{N}$-Butyl- $\alpha$-hydroxybenzenethioacetamide (8a). Isolation was performed by HPLC then PTLC $\left({ }^{n}\right.$ hexane/Et $\left.{ }_{2} \mathrm{O}=4 / 1\right)$. Mp 67.0-68.0 ${ }^{\circ} \mathrm{C} ;{ }^{1} \mathrm{H}$ NMR $\left(270 \mathrm{MHz}, \mathrm{CDCl}_{3}\right) \delta 0.89$ (t, $J=7.4 \mathrm{~Hz}, 3 \mathrm{H}$ ), 1.29 (sextet, $J=7.4 \mathrm{~Hz}, 2 \mathrm{H}$ ), 1.57 (quint, $J=7.4 \mathrm{~Hz}, 2 \mathrm{H}$ ), 3.62 (q, $J=7.4 \mathrm{~Hz}, 2 \mathrm{H}), 4.31(\mathrm{~s}, 1 \mathrm{H}), 5.14(\mathrm{~d}, J=2.9 \mathrm{~Hz}, 1 \mathrm{H}), 7.34-7.38(\mathrm{~m}, 5 \mathrm{H}), 7.82(\mathrm{~s}, 1$ $\mathrm{H}) ;{ }^{13} \mathrm{C} \mathrm{NMR}\left(68 \mathrm{MHz}, \mathrm{CDCl}_{3}\right) \delta 13.6,20.0,29.8,45.6,78.5,126.9,128.7,128.8$, 140.4, 203.0; IR (KBr) 3286, 3192, 1560, $996 \mathrm{~cm}^{-1} ; \mathrm{MS} \mathrm{m} / \mathrm{z}$ (relative intensity) $223\left(\mathrm{M}^{+}\right.$, 24), 117 (32), 116 (16), 107 (27), 84 (57), 77 (45); HRMS calcd for $\mathrm{C}_{12} \mathrm{H}_{17} \mathrm{NOS} 223$. 1031 , found 223.1040 .

$N$-Butyl- $\alpha$-hydroxy- $\alpha, \alpha$-pentamethylenethioacetamide (8b). Isolation was performed by HPLC then PTLC $\left({ }^{n}{ }^{h}\right.$ exane/ $\left./ \mathrm{Et}_{2} \mathrm{O}=4 / 1\right)$. Mp 116.0-117.0 ${ }^{\circ} \mathrm{C} ;{ }^{1} \mathrm{H}$ NMR $\left(270 \mathrm{MHz}, \mathrm{CDCl}_{3}\right) \delta 0.96(\mathrm{t}, J=7.3 \mathrm{~Hz}, 3 \mathrm{H}), 1.26-1.73(\mathrm{~m}, 12 \mathrm{H}), 2.33(\mathrm{td}, J=14.7$, $4.4 \mathrm{~Hz}, 2 \mathrm{H}), 2.43$ (s, $1 \mathrm{H}), 3.64$ (td, $J=7.3,9.3 \mathrm{~Hz}, 2 \mathrm{H}), 8.73(\mathrm{~s}, 1 \mathrm{H}) ;{ }^{13} \mathrm{C}$ NMR $(68$ $\left.\mathrm{MHz}, \mathrm{CDCl}_{3}\right) . \delta 13.7,20.1,21.7,24.7,30.0,36.9,45.4,80.5,208.0$; IR (KBr) 3300, 3261, 2960, 2946, 2928, 1538, $967 \mathrm{~cm}^{-1}$; MS m/z (relative intensity) $215\left(\mathrm{M}^{+}, 100\right), 117$ (59), 84 (70); HRMS calcd for $\mathrm{C}_{11} \mathrm{H}_{21}$ NOS 215.1344, found 215.1342.

$\mathrm{N}$-Butylcarboanilidethioformamide (8c). Isolation was performed by HPLC. Mp 50.0-52.0 ${ }^{\circ} \mathrm{C}$; ${ }^{1} \mathrm{H}$ NMR $\left(270 \mathrm{MHz}, \mathrm{CDCl}_{3}\right) . \delta 0.98(\mathrm{t}, J=7.4 \mathrm{~Hz}, 3 \mathrm{H}), 1.45$ (sextet, $J=$ $7.4 \mathrm{~Hz}, 2 \mathrm{H}$ ), 1.73 (quint, $J=7.4 \mathrm{~Hz}, 2 \mathrm{H}$ ), 3.71 (q, $J=7.4 \mathrm{~Hz}, 2 \mathrm{H}$ ), 7.19 (t, $J=7.6 \mathrm{~Hz}$, 
$1 \mathrm{H}), 7.38(\mathrm{t}, J=7.6 \mathrm{~Hz}, 2 \mathrm{H}), 7.65(\mathrm{~d}, J=7.6 \mathrm{~Hz}, 2 \mathrm{H}), 9.58(\mathrm{~s}, 1 \mathrm{H}), 10.21(\mathrm{~s}, 1 \mathrm{H})$; ${ }^{13} \mathrm{C}$ NMR $\left(68 \mathrm{MHz}, \mathrm{CDCl}_{3}\right) \delta 13.7,20.2,29.7,46.3,119.8,125.4,129.2,136.7,156.0$, 185.9; IR (KBr) 3283, 3232, 2953, 1674, 1602, 1535, 1446, 753, $704 \mathrm{~cm}^{-1} ; \mathrm{MS} \mathrm{m} / \mathrm{z}$ (relative intensity) $236\left(\mathrm{M}^{+}, 100\right), 193$ (19), 93 (31), 57 (36); HRMS calcd for $\mathrm{C}_{12} \mathrm{H}_{16} \mathrm{~N}_{2} \mathrm{OS} 236.0983$, found 236.0991.

$\mathrm{N}$-Butylbenzoylthioformamide (8d). Isolation was performed by PTLC $\left({ }^{n}\right.$ hexane/Et $\left.{ }_{2} \mathrm{O}=4 / 1\right)$ then HPLC; Mp 72.5-73.5 ${ }^{\circ} \mathrm{C} ;{ }^{1} \mathrm{H}$ NMR $\left(270 \mathrm{MHz}, \mathrm{CDCl}_{3}\right) \delta 1.00$ (t, $J=7.4 \mathrm{~Hz}, 3 \mathrm{H}$ ), 1.47 (sextet, $J=7.4 \mathrm{~Hz}, 2 \mathrm{H}$ ), 1.76 (quint, $J=7.4 \mathrm{~Hz}, 2 \mathrm{H}$ ), 3.78 (q, $J=7.4 \mathrm{~Hz}, 2 \mathrm{H}), 7.44$ (t, $J=7.8 \mathrm{~Hz}, 2 \mathrm{H}), 7.58$ (t. $J=7.8 \mathrm{~Hz}, 1 \mathrm{H}), 8.05$ (d, $J=7.8 \mathrm{~Hz}$, $2 \mathrm{H}), 8.27(\mathrm{~s}, 1 \mathrm{H}) ;{ }^{13} \mathrm{C}$ NMR $\left(68 \mathrm{MHz}, \mathrm{CDCl}_{3}\right) \delta 13.7,20.2,29.9,45.1,128.1,130.8$, 133.7, 133.9, 187.6, 193.3; IR (KBr) 3261, 1649, 1538, 1274, 1263, $700 \mathrm{~cm}^{-1} ; \mathrm{MS} \mathrm{m} / \mathrm{z}$ (relative intensity) $221\left(\mathrm{M}^{+}, 79\right), 116$ (50), 105 (100), 77 (53), 72 (44), 57 (91); HRMS calcd for $\mathrm{C}_{12} \mathrm{H}_{15} \mathrm{NOS} 221.0874$, found 221.0881.

$N$-Phenyl- $\alpha$-hydroxybenzenethioacetamide (8e). Isolation was performed by HPLC. Mp 109.5-110.5 ${ }^{\circ} \mathrm{C} ;{ }^{1} \mathrm{H}$ NMR $\left(270 \mathrm{MHz}, \mathrm{CDCl}_{3}\right) \delta 4.25(\mathrm{~s}, 1 \mathrm{H}), 5.31$ (d, J=3.4 Hz, 1 H), 7.27 (t, $J=7.3 \mathrm{~Hz}, 1 \mathrm{H}), 7.34-7.40(\mathrm{~m}, 5 \mathrm{H}), 7.44-7.48(\mathrm{~m}, 2 \mathrm{H}), 7.68(\mathrm{~d}, J=7.3 \mathrm{~Hz}$, $2 \mathrm{H}), 9.43(\mathrm{~s}, 1 \mathrm{H}) ;{ }^{13} \mathrm{C} \mathrm{NMR}\left(68 \mathrm{MHz}, \mathrm{CDCl}_{3}\right) \delta 79.8,123.0,123.0,127.1,127.1,129.0$, 129.0, 137.8, 140.3, 201.2; IR (KBr) 3248, 1598, 1547, 1492, 1402, 1376, 1009, 767, $700 \mathrm{~cm}^{-1} ; \mathrm{MS} \mathrm{m} / \mathrm{z}$ (relative intensity) $243\left(\mathrm{M}^{+}, 66\right), 136(100), 135$ (85), 107 (31), 77 (48). Anal. Calcd for $\mathrm{C}_{14} \mathrm{H}_{13} \mathrm{NOS}$ : C, 69.10; H, 5.38; N, 5.76; S, 13.18. Found: C, 68.81; H, 5.47; N, 5.70; S, 13.23.

\section{6) References}

S1) Engman, L.; Cava, M. P. Synth. Commun. 1982, 12, 163.

S2) Kronenthal, D. R.; Han, C. Y.; Taylor, M. K. J. Org. Chem. 1982, 47, 2765.

S3) Iriuchijima, S.; Maniwa, K.; Tsuchihashi, G. J. Am. Chem. Soc. 1975, 97, 596. 